\title{
The rate, reasons and predictors of hospital discharge against medical advice among inpatients of a tertiary health facility in North-central Nigeria
}

\author{
Samuel Adebowale Adefemi ${ }^{1,2}$, Ibrahim Taiwo Adeleke ${ }^{2,3,4}$, Philip Gara ${ }^{1}$, \\ Olanrewaju Oloundare Abdul Ghaney ${ }^{3}$, Sunday Akingbola Omokanye ${ }^{2,3,4}$, \\ Aivonya Momoh Jimoh Yusuf ${ }^{3,4}$ \\ ${ }^{1}$ Department of Family Medicine, Federal Medical Centre, Bida, Nigeria \\ ${ }^{2}$ Centre for Health \& Allied Researches, Bida, Nigeria \\ ${ }^{3}$ Department of Health Information, Federal Medical Centre, Bida, Nigeria \\ ${ }^{4}$ Health Informatics Research Initiatives in Nigeria, Bida, Nigeria
}

\section{Email address:}

samueladefemi@yahoo.com (S. A. Adefemi),ibratadeleke_aliseyin@yahoo.com (I. T. Adeleke), garaphils@yahoo.com (P. Gara), abdulghanneeyy@yahoo.com (O. O. A. Ghaney), sunjac4jesus@yahoo.com (S. A. Omokanye), aivonya38@yahoo.com (A. M. J. Yusuf)

\section{To cite this article:}

Samuel Adebowale Adefemi, Ibrahim Taiwo Adeleke, Philip Gara, Olanrewaju Oloundare Abdul Ghaney, Sunday Akingbola Omokanye, Aivonya Momoh Jimoh Yusuf. The Rate, Reasons and Predictors of Hospital Discharge against Medical Advice among Inpatients of a Tertiary Health Facility in North-Central Nigeria. American Journal of Health Research. Special Issue: Health Information Technology in Developing Nations: Challenges and Prospects Health Information Technology. Vol. 3, No. 1-1, 2014, pp. 11-16.

doi: 10.11648/j.ajhr.s.2015030101.12

\begin{abstract}
Objective: To determine the rate of hospital discharges against medical advice (DAMA), identify reasons for such discharges and identify factors predicting such discharges compared with controls. Methods: This is a retrospective health records review of all patients who discharged against medical advice and matched cases of those discharged normally between 2009 and 2011. Patients with DAMA were identified and relevant socio-demographic as well as clinical data were extracted from their clinical charts. We compared characteristics of case control patients using $\chi 2$ and Wilcoxon Rank Sum test as appropriate. We used multivariate logistic regression to model the correlates of discharged against medical advice. We included in the analysis each characteristics that was significant $(p=0.05)$ in bivariate comparison. We report odds ratio (ORs) and confidence intervals (CIs) from this model. Results: The prevalence of discharged against medical advice was $0.72 \%$ over the three year period. Patients who discharged against medical advice were younger, male gender and students. In addition they were predominantly Muslims and married with no form of formal education. Furthermore, residence in Bida where the hospital is situated and payment out of pocket is associated with DAMA. Most of these patients DAMA within the first five days of admission and signatories to the discharge were mostly parents, spouse, and other family members. Reasons for DAMA include financial constraints, improved health and desire to seek other treatments (faith/traditional based). The male gender, students and those without formal education all conferred increase odds of DAMA, while persons in age range 21-30 years, and married conferred protection against DAMA. Furthermore, individuals with normal delivery (SVD) (18), followed by RTA with minor injury such as bruises/laceration (14), and RTA with fracture (12) were much more likely to DAMA. Conclusion: Comparatively, DAMA rate at Federal Medical Centre, Bida is low. The factors associated with such DAMA include younger age, male sex and being a student. In addition, residing in Bida town and payments for healthcare out of pocket increase the likelihood of DAMA. We recommend formulation of explicit DAMA guidelines for the hospital and strengthening of the existing National Health Insurance Scheme as panacea for reduction in the rate of DAMA.
\end{abstract}

Keywords: Discharge against Medical Advice, Health Records Review, Predictive Factors, Reasons, Tertiary Hospital, Rate

\section{Introduction}

Patients have the right to discharge against medical advice
(DAMA) based on human rights and the 'Patients' charter (right to self-determination or autonomy), while the healthcare providers also have the right to do what they think is best for 
the patient (to act with beneficence) [1]. This ethical problem is often a source of frustration for the medical personnel, who has to maintain a balance between the interests of the patient in preserving his/her autonomy and the interests of the state in maintaining life and adequate health [2].

Discharge against medical advice arises when a patient or his or her carer decides to leave the hospital before the treating physician recommends they do so [3]. Discharge against medical advice is of concern because it is assumed that these patients are leaving too soon and that adverse consequences may follow. Patients do these for a variety of reasons. In the study by Baptist et al [4] for instance, the reasons for DAMA were identified following a review of health records of asthma patients who left against medical advice and these included: dissatisfaction with care; patient was feeling better; and family obligations, while other studies [5-6] cited financial obligations and legal issues in addition to the above reasons.

Generally speaking, depending on the patient population and the type of treatment, the rate of DAMA has varied widely, ranging from $0.7 \%$ to $2.2 \%$ for general medical admissions, $6 \%$ to $54 \%$ for psychiatric admissions [7] and $0.9 \%$ for emergency admissions [8], and as such, care must be taken in its interpretation. For example, some chronic medical problems like diabetes, hypertension (which ordinarily, with best of treatments is only controlled), tend to require longer time of care than acute conditions like malaria. Whereas, patients tend to interpret a shorter length of stay as favourable outcome. The predictors of DAMA from previous studies include younger age, male sex, substance abuse disorders, lack of a personal physician and lack of health insurance [9]. Those with prior discharge against medical advice have been identified in previous studies [5, 10-12] as risk factors for discharge against medical advice. Regrettably however, disease severity have not been found as a predictor of this repulsive but permitted practice.

Discharge against medical advice may expose the patient to risk of an inadequately treated medical problem and results in the need for readmission and prolonged morbidity. In fact, a study [3] found about $32 \%$ of the patients was readmitted within 30 days, whereas only $12 \%$ of regular discharges led to readmission within 30 days. Furthermore, patients discharged against medical advice were more likely to have a subsequent admission for the same (or a related) diagnosis in the subsequent month $(28 \%$ v. $8 \%)$ and to have longer stays in hospital for any readmission (median 5 v. 0 days) [7]. In addition, the healthcare provider-patient relationship may be strained in the event that the patient had to be readmitted. Understanding why patients choose to leave the hospital against medical advice has obvious importance because of the potential to identify those at higher risk and therefore intervene earlier to prevent excess morbidity, mortality, and reduce healthcare costs [3].

\subsection{Aim of the study}

This study attempted to determine the rate of hospital discharges against medical advice at Federal Medical Centre,
Bida, identify reasons for such discharges and identify factors predicting such discharges compared with controls.

\section{Methods}

\subsection{Study Site}

Federal Medical Centre, Bida is a tertiary health institution and a referral centre that provides specialty care in the fields of Medicine, Surgery, Psychiatry, and Obstetrics \& Gynaecology among others.

\subsection{Sample Selection}

This is a retrospective review of health records of all patients who discharge against medical advice and matched cases of those discharged normally between 2009 and 2011 in the hospital. We defined patients as discharged against medical advice (DAMA) if they signed acceptable hospital clinical notes (e.g. progress notes) acknowledging that they were discharging themselves contrary to the advice of their physician as against those who absconded. Controls are cases that were discharged normally on the advice of the attending physician. We identified a total of 27,136 hospital discharges from 2009-2011. We excluded 2,128 patients who died in the hospital. Of the remaining 25,008 discharges, $179(0.72 \%)$ of these patients accounted for discharged against medical advice. We selected $1.5 \%$ [13] of the remaining 24,829 hospital discharges at random to create a control group of 372 patients representing 372 discharges with physician approval. To prevent double counting, especially of those patients with multiple admissions, a single index discharge was selected at random for these patients. We chose the individual patient rather than the hospital discharge as the unit of analysis to determine those who are particularly vulnerable to be discharged against medical advice. Cases and controls were matched for their date of admission or the closest admissions possible.

\subsection{Data Elements}

A data extraction form (as designed by the authors) was used to collect patient information from the patient charts. The design ensured de-identification of the patients' health records and as such, no data element can be traced to any patient now or in future [14-15]. Data on age, gender, other demographic characteristics and chief diagnosis were extracted. In addition, the admission and discharge rate using standard classification criteria, the International Statistical Classification of Diseases and Related Health Problems $10^{\text {th }}$ Revision (ICD-10), and code for the patient's principal discharge diagnosis were assigned by health information management (HIM) professionals among the authors, after chart abstraction. We aggregated continuous diagnosis codes in appropriate cases into higher order diagnoses.

\subsection{Inclusion and Exclusion Criteria}

For patients, who DAMA more than once during the study 
period, only the first time DAMA was included. In addition, patients who received treatment but did not require admission were excluded. Patients who disappeared after registration were also not included. For the purpose of this study, admissions and discharge data covering departments/units such as ophthalmology, ENT, orthopaedic, and the neonatal intensive care, were added to that of their parent departments, i.e., Surgery and Paediatrics respectively.

\subsection{Statistical Analysis}

We compared characteristics of case control patients using $\chi 2$ and Wilcoxon Rank Sum test as appropriate. We used multivariate logistic regression to model the correlates of discharged against medical advice. We included in the analysis each characteristic that was significant $(p=0.05)$ in bivariate comparison. We report odds ratio (ORs) and confidence intervals (CIs) from this model.

\subsection{Ethics}

The Hospital Research Ethics Committee of the hospital gave approval for the conduct of this study. This approval covers patients' consent as this consent was difficult to obtain from individual patient [16].

\section{Results}

The prevalence of discharged against medical advice was $0.72 \%$ over the three year (2009-2011) period.

Table 1. Selected patient characteristics of those discharged against medical advice

\begin{tabular}{|c|c|c|c|c|}
\hline Patient characteristics & DAMA $(n=179)$ & Discharge with approval $(n=372)$ & $\chi^{2}$ & $p$-value \\
\hline \multicolumn{5}{|l|}{ Age } \\
\hline$<10$ & 36 & 142 & 37.27 & 0.000 \\
\hline $11-20$ & 32 & 61 & & \\
\hline $21-30$ & 44 & 40 & & \\
\hline $31-40$ & 33 & 48 & & \\
\hline $41-50$ & 12 & 33 & & \\
\hline $51-60$ & 13 & 13 & & \\
\hline \multicolumn{5}{|l|}{ Sex } \\
\hline Male & 90 & 139 & 8.30 & 0.004 \\
\hline Female & 89 & 233 & & \\
\hline \multicolumn{5}{|l|}{ Occupation } \\
\hline Civil service & 17 & 42 & 46.91 & 0.000 \\
\hline Unemployed & 33 & 46 & & \\
\hline Farming & 26 & 20 & & \\
\hline Driving & 6 & 11 & & \\
\hline Schooling & 36 & 79 & & \\
\hline Children & 32 & 124 & & \\
\hline Others & 15 & 4 & & \\
\hline \multicolumn{5}{|l|}{ Religion } \\
\hline Christianity & 33 & 103 & 5.57 & 0.018 \\
\hline Islam & 146 & 269 & & \\
\hline \multicolumn{5}{|l|}{ Marital status } \\
\hline Married & 99 & 158 & 8.70 & 0.003 \\
\hline Single & 79 & 214 & & \\
\hline Not indicated & 1 & 0 & & \\
\hline \multicolumn{5}{|l|}{ Educational level } \\
\hline None & 93 & 197 & 14.25 & 0.014 \\
\hline Completed primary & 20 & 57 & & \\
\hline Completed secondary & 21 & 59 & & \\
\hline Completed tertiary & 19 & 38 & & \\
\hline \multicolumn{5}{|l|}{ Place of residence } \\
\hline Bida & 75 & 166 & 10.94 & 0.012 \\
\hline Minna & 13 & 20 & & \\
\hline Other LGs* within Niger state & 57 & 79 & & \\
\hline Other states & 34 & 107 & & \\
\hline
\end{tabular}

*LGs $=$ Local Governments

Table 1 above reveals that compared to controls (patients discharged with physician's approval), most patients who discharged against medical advice were younger, male gender, unlettered Muslims, married and students. Furthermore, residence in Bida where the hospital is situated and payment out of pocket is associated with DAMA.

Common principal diagnosis in DAMA patients include normal SVD $(n=18)$, RTA with minor injury $(n=14)$ and RTA with fracture $(\mathrm{n}=12)$. 
Table 2. Hospital stay and signatories to DAMA

\begin{tabular}{ll}
\hline Length of stay in hospital (in days) & Frequency (\%) \\
\hline $1-5$ & $87(48.6)$ \\
$6-10$ & $37(20.7)$ \\
$11-15$ & $23(12.8)$ \\
$6-30$ & $24(13.4)$ \\
$>30$ & $8(4.5)$ \\
Signatories to DAMA & \\
Spouse & $45(25.1)$ \\
Children & $11(6.1)$ \\
Parents & $58(32.4)$ \\
Relations & $33(18.4)$ \\
Friends & $4(2.2)$ \\
Patient themselves & $28(15.6)$ \\
\hline
\end{tabular}

Most of the DAMA patients $(87,48.6 \%)$ initiated their discharge within the first 5 days of admission and signatories to the discharge are mostly parents, spouse, and relations

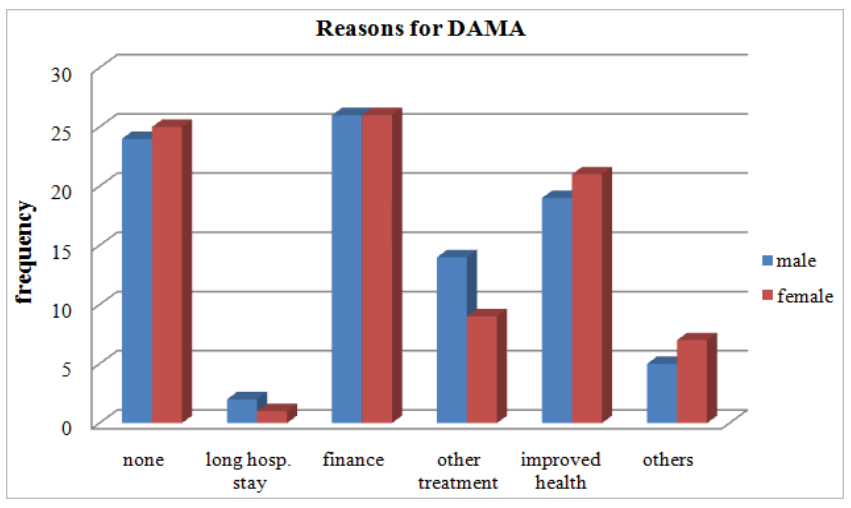

Fig 1. Reasons for DAMA

Reasons for DAMA include financial constraints (50, $27.9 \%)$, improved health $(40,22.3 \%)$ and desire to seek other treatments (faith/traditional based) $(23,12.8 \%)$.

Table 3. Multivariate analysis of DAMA cases at FMC Bida, 2009-2011

\begin{tabular}{ll}
\hline Variable & Odds Ratio (95\% CI) \\
\hline Age & $0.82(0.5-1.3)$ \\
Male gender & $2.94(0.6-13.9)$ \\
Occupation & $1.22(1.0-1.5)$ \\
Marital status & $0.41(0.1-1.7)$ \\
Education level & $0.76(0.2-2.8)$ \\
Place of residence & $1.58(1.1-2.4)$ \\
Duration of hospital stay before DAMA & $1.07(0.8-1.4)$ \\
\hline
\end{tabular}

The results of the multivariate analysis (Table 3) shows that male gender, students and those without formal education all conferred increase odds of DAMA, while persons in age range 21-30 years, and the married conferred protection against DAMA. Furthermore, individuals with SVD (Normal delivery - 18), followed by RTA with minor injury (bruises/laceration -14), and RTA with fracture - 12 were much more likely to DAMA.

\section{Discussion}

Patients have right to decide to leave hospital [1, 12], however this decision if taken against medical advice is not without consequences. The overall DAMA rate of $0.72 \%$ at FMC Bida, although higher, is comparable to the $0.34 \%$ reported by Duno et al [17], $0.57 \%$ reported by Moyse et al [18], but differs markedly from the range reported elsewhere in Africa (1.2\%-12\%) [19-20]. This may be explained by the similarity of the study population of this survey to that in the study by Duno et al [17] since both comprised general medical admissions excluding psychiatric and Accident and Emergency patients, or paediatric admissions who are known to have higher prevalence of DAMA [7]. By contrast, Alebiosu and Raimi [19], in a 2-year study of DAMA at a teaching hospital in South-western Nigeria, reported a DAMA rate of $2.8 \%$, apparently due to their inclusion of accident and emergency patients, who accounted for $45.2 \%$ of all DAMA, in their study.

Patients who leave the hospital against physician orders have been reported to be at a higher risk of adverse health outcomes and have increased hospital readmission rates compared with patients who follow physician advice. The readmission rate among DAMA in this study was 3.9\%. This is probably underestimated since only seven persons who felt that their health had not improved came back to our hospital within two weeks of DAMA. Other possible reasons for this low rate may include reasons for DAMA in the first place i.e. funding as most of the payments for treatment are out of pocket. The preponderance of male gender who DAMA is also consistent with similar studies in the country and elsewhere $[6,10,13,21-22]$. The fact that up to $40(22.3 \%)$ patients felt their condition had improved and saw no reason of staying longer in the hospital, probably suggest we might review our hospital discharge policy so that patients are not kept beyond necessary as patients achieve best healing where there is less disruption from their familiar environment. A closer look at the findings from our study revealed that majority of index cases were normal SVD, who were not primipara, and RTA patients who had minor/trivial injury. The finding that majority of DAMA left within the first fve days on admission is consistent with similar studies from Nigeria [23].

We found that important correlates of DAMA were male gender, being a student and persons with no formal education. Financial constraints, improvement in health, and wanting to seek other treatments accounted for majority of DAMA patients. The prevailing harsh economic environment in Nigeria, and the limited coverage of the National Health Insurance Scheme (only formal sector with few localised community schemes), with its expected impact on individuals' healthcare financing, partly explains this $[3,6]$.

\subsection{Study Limitations}

This study had several limitations. As a single institutional study, its generalizability may be limited to similar hospitals. We also could not identify patient who DAMA and later were 
readmitted to another hospital or patient who left our hospital with physician approval after DAMA elsewhere. Furthermore, our analysis is restricted to the patient chief diagnosis code and may have underestimated the number of patient with comorbid conditions as reported in earlier studies [24-25]. Unlike most studies that associate DAMA among those of unsound mind (psychiatric illnesses) [26], our series only had one such person whose primary diagnosis is schizophrenia. Perhaps their finding may not be unconnected to the setting of their study which is either emergency room setting or specific speciality compared with this study that looked at the hospital as a whole.

\section{Conclusion}

Comparatively, DAMA rate at Federal Medical Centre, Bida is low. The factors associated with such DAMA include younger age, male sex and being a student. Moreover, residing in Bida town and payments for healthcare out of pocket increase the likelihood of DAMA. We recommend that formulation of explicit DAMA guidelines and strengthening of the existing National Health Insurance Scheme as panacea for reduction in the rate of DAMA.

\section{Acknowledgment}

The authors wish to thank Mr. J. O. Lawal of the Department of Health Information, for his assistance during health records review and data abstraction. Also, Mr. D Aliyu of the Department of Nusring Services, Ahmadu Bello University Teaching Hospital, Zaria for his comments on the final manuscript.

\section{References}

[1] T. W. Stern, B. C. Silverman, A. S. Smith, T. A. Stern. Prior discharge against medical advice and withdrawal of consent: what they can teach us about patient management. Prim. Care Companion CNS Disord. 2011;13(1):doi:10.4088/PCC.10f01047b/u.

[2] J. T. Berger. Discharge against medical advice: ethical considerations and professional obligations. J Hosp Med. 2008;3:403e8.

[3] D. J. Alfandre. "I'm going home": Discharges against medical advice. Mayo Clinic Proceedings. 2009;84:255-260.

[4] A. P. Baptist, I. Warrier, R. Arora, J. Ager, R. M. Massanari. Hospitalized patients with asthma who leave against medical advice: characteristics, reasons, and outcomes. J Allergy Clin Immunol. 2007;19(4):924-929.

[5] P. Green, D. Watts, S. Poole, V. Dhopesh. Why patients sign out against medical advice (AMA): factors motivating patients to sign out AMA. Am J Drug Alcohol Abuse. 2004;30(2):489493.

[6] Z. Y. Aliyu. Discharge against medical advice: sociodemographic, clinical and financial perspectives. Int J Clin Pract. 2002;56(5):325-327.
[7] A. H. Anis, H. Sun, D. P. Guh, A. Palepu, M. T. Schechter, M. V. O'shaughnessy. Leaving hospital against medical advice among HIV-positive patients. CMAJ. 2002;167:633-637.

[8] T. W. Wong, K. M. Lee, R. Chan, C. C. Lau. A study of patients who leave an accident and emergency department against medical advice. Hong Kong Journal of Emergency Medicine. 2000;7(1): 22-26.

[9] R. Saitz. Discharges against medical advice: time to address the causes. CMAJ. 2002;167(6):647-648.

[10] S. A. Ibrahim, C. K. Kwoh, E. Krishnan. Factors associated with patients who leave acute-care hospitals against medical advice. Am J Public Health. 2007;97(12):2204-2208. Epub 2007 Oct 30.

[11] S. W. Hwang, J. Li, R. Gupta, V. Chien, R. E. Martin. What happens to patients who leave hospital against medical advice? CMAJ. 2003;168(4):417-420.

[12] S. A. A. Abhari, M. M. Abbasi, N. P. Jafar, H. Zerrati. Factors associated with premature discharge of patients with psychosis against medical advice. Iran J Psychiatry. 2006;27-30.

[13] S. N. Weingart, R. B. Davis, R. S. Phillips. Patients discharged against medical advice from a general medicine service. J Gen Intern Med. 1998;13:568-571.

[14] I. T. Adeleke, A. O. Adekanye, K. A. Onawola, et al. Data quality assessment in healthcare: a 365-day chart review of inpatients' health records at a Nigerian tertiary hospital. J Am Med Inform Assoc. 2012;19:1039-1042. doi:10.1136/amiajnl2012-000823.

[15] L. A. Rinehart-Thompson. Legal issues in health information management technology. In: Johns ML, ed. Health Information Management Technology: An Applied Approach. 2nd edn. Chicago: American Health Information Management Association, 2007:704e5.

[16] Doyal L. Informed consent in medical research: journals should not publish research to which patients have not given fully informed consentewith three exceptions. BMJ 1997;314:1107e11.

[17] R. Duno, E. Pousa, J. Sans, C. Tolosa, A. Ruiz. Discharge against medical advice at a general hospital in Catalonia. Gen Hosp Psychiatry. 2003;25:46-50.

[18] H. S. Moyse, W. E. Osmun. Discharge against medical advice: a community hospital experience. Can. J. Rural Med. 2004;9(3):148-153.

[19] C. O. Alebiosu, T. H. Raimi. A study of hospital patients' discharge against medical advice in the Ogun State University Teaching Hospital, Sagamu, Nigeria. Nigeria Medical Practitioner. 2001;40:33-35.

[20] A. N. Onyiruka. Discharge of hospitalised under-fives against medical advice in Benin City, Nigeria. Nig J Clin Pract. 2007;10:200-204.

[21] P. J. Devitt, A. C. Devitt, A. Dewan. Does identifying a discharge as "against medical advice" confer legal protection? J Fam Pract. 2000;4:224-227.

[22] N. E. Ngim, T. Nottidge, A. Oku, A. F. Akpan. Why do orthopaedic trauma patients leave hospital against medical advice? Ibom Medical Journal 2013;6(1):1-4. 
[23] A. M. Udosen, E. Glen, S. Ogbudu, E. Nkposong. Incidence of leaving against medical advice (LAMA) among patient admitted at the accident and emergency unit of the University of Calabar Teaching Hospital, Calabar, Nigeria. Nigeria Journal of Clinical Practice 2006;9(2):120-123.

[24] B. Eze, K. Agu, J. Nwosu. Discharge against medical advice at a tertiary centre in South-eastern Nigeria: sociodemographic and clinical dimensions. Patient Intelligence. 2010:2:27-31.
[25] L. R. Ariede. Implementation of the National Health Insurance Scheme: the dawn of a new era in healthcare financing in Nigeria? Sahel Med J. 2003;6:1-5.

[26] E. Moy, B. A. Bartman. Race and hospital discharge against medical advice. Journal of National Medical Association. 1996;88:658-660. 